\title{
Kinetic and Morphological Analysis of $C$. necator Cells During the Synthesis and Degradation of Intracellular Polyhydroxyalkanoates (PHAs).
}

\author{
Daiana Nygaard $^{1 *}$, Oxana Yashchuk ${ }^{1,2}$, Ana Heidenreich ${ }^{1}$ and Élida Hermida ${ }^{1,2}$. \\ 1. School of Science and Technology, National University of San Martín (UNSAM), Av. 25 de mayo \\ 1147 (B1650HMK), San Martín, Buenos Aires, Argentina. \\ 2. Argentine Council of Scientific and Technical Research (CONICET), Godoy Cruz 2290 \\ (C1425FQB), CABA - Argentina. \\ *Corresponding author: dnygaard@unsam.edu.ar
}

The increasing commercialization of PHAs is due to the excellent combination of their properties: thermoplasticity, biodegradability and biocompatibility, which allow the design of products for diverse applications [1], [2]. PHAs are accumulated by microorganisms in the form of intracellular granules under conditions of nutritional stress [3]. The aim of this work is the study by Transmission Electron Microscopy (TEM) the formation, growth and degradation of the PHAs granules during bacteria fermentation.

The study was carried out in three stages. In stage 0 , the inoculum of $C$. necator in TYF medium (Tryptone, Yeast, Fructose) was cultivated during $24 \mathrm{~h}$. In stage 1, washed cells were incubated during $72 \mathrm{~h}$ in a medium statistically optimized for the PHB production [4]. Finally, in stage 2, cells were incubated for $98 \mathrm{~h}$ in a carbon-free medium such that $C$. necator degrades PHB. Thus, the whole incubation of stages 1 and 2 lasted $170 \mathrm{~h}$ (Fig. 1).

In stage $0, C$. necator synthesized from 1 to 6 spherical PHA granules/cell with a maximum area of $0.18 \mu^{2}$. After $24 \mathrm{~h}$ of stage 1, some cells grew up to 3-times their length and 5-times their area, with a variable granule content up to 17. Furthermore, TEM images favored the "budding model" [5], in which nascent granules are located adjacent to or even bound to the inner side of the cytoplasmic membrane (Fig. 2). Then, a granule coalescence was observed, leading even to a single granule of 0.43 $\mu \mathrm{m}^{2}$. Finally, the TEM micrographs clearly showed the granules degradation and the cell size decrease along Stage 2.

In conclusion, TEM allowed studying the kinetics and morphology of the different stages of accumulation and degradation of the PHAs granules, essential issues for scaling up the production of these biodegradable polyesters. 


\section{References:}

[1] A.K. Singh et al., Applied Microbiology and Biotechnology 37 (2019), p. 1-26.

[2] M. Koller, Molecules 23 (2018), p. 1-20.

[3] S. Khanna and A.K. Srivastava, Process Biochemistry 40 (2005), p. 607-619.

[4] D. Nygaard et al., Heliyon 5 (2019), p. 1-12.

[5] N. Thomson et al., Soft Matter 6 (2010), p. 4045-4057.

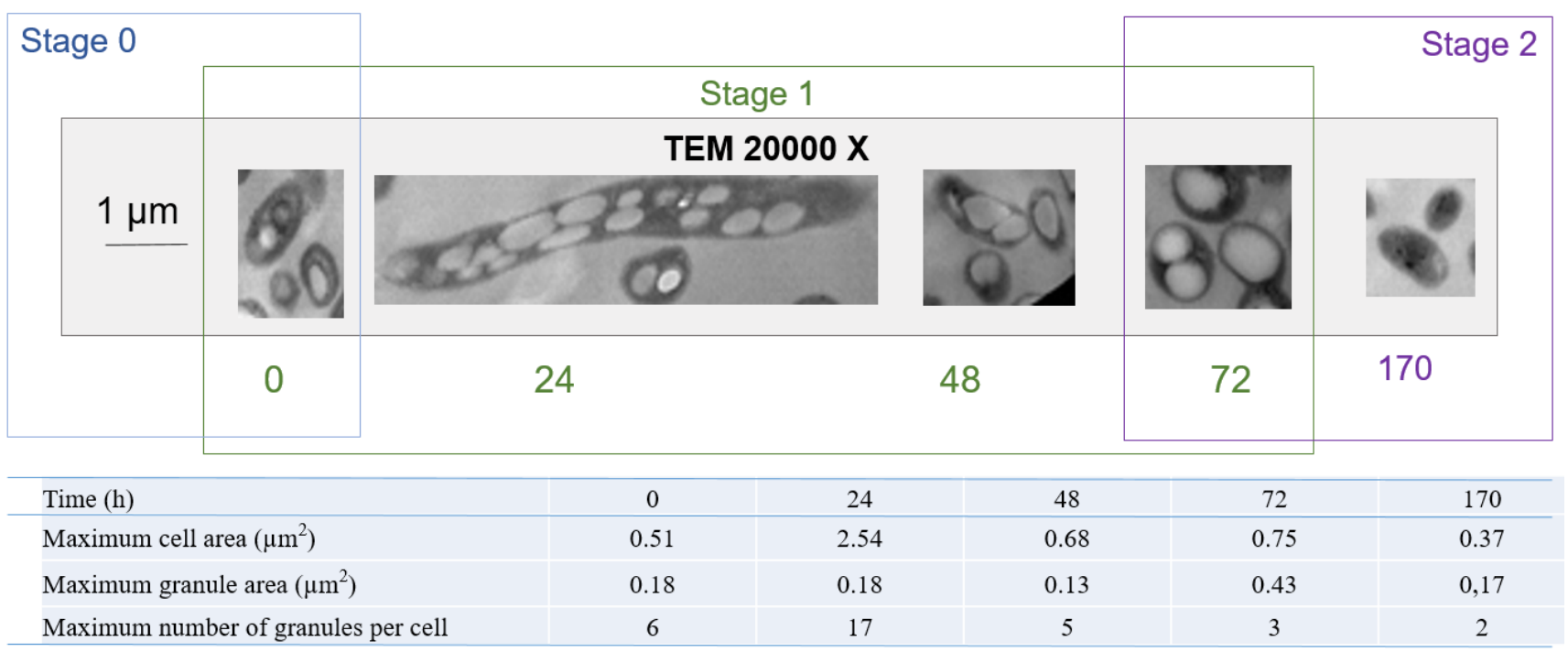

Figure 1: TEM micrographs of cells obtained in different fermentation stages with theirs corresponding areas $(20000 \mathrm{X})$;

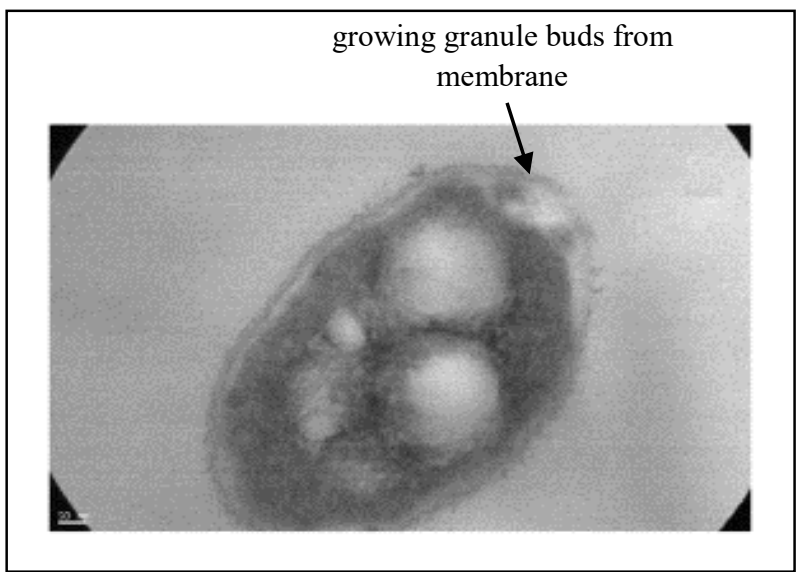

Figure 2: TEM image of a cell favored the "budding model" (140 $000 \mathrm{X})$. 